\title{
Analysis on Wushu Sanda of Zhang Kaiyin wining factor
}

\author{
Hongbo Zhao ${ }^{1, \text { a }}$ \\ ${ }^{1}$ Luoyang Normal College, Henan, China \\ a327040475@qq.com
}

Keywords: Sanda Competition; Zhang Kaiyin; winning

\begin{abstract}
The flexible use of a variety of boxing, kicking and the skills in competition are the basic reasons for Zhang Kaiyin to win the championship. His successive aggressive martial spirit, confident and calm challenge attitude, unyielding faith after twists and turns, and he is good at grasping the opportunity in the changing environment, these are primary causes for his victory. The analysis on Zhang Kaiyin's wining factors of Martial Arts, which provides a reference for the study of Chinese Martial Arts theory.
\end{abstract}

\section{Introduction}

Sanda.Sanda is a main manifestation of Chinese martial arts, it is also called "Sanshou", and called as "Xiangbo", "Shoubo", and "Jiji”, etc. Sanda is a kind of competitive sport, in which two people carry out unarmed combat with kicking, beating, falling, and the corresponding techniques and tactics of martial arts in accordance with the competition rules. As part of the cultural heritage of the Chinese nation, Sanda is popular among the people, and it has a profound mass basis.

Zhang Kaiyin has brilliant records before the championship in the Free Fighting Competition. Zhang is the present excellent free fighting athlete in our country, over 10 years of Sanda career enriches Zhang Kaiyin's competition experience and his technical characteristics has a typical value of reference. Reviewing his brilliant martial arts history, he started to learn martial arts from the age of 13. In 2003, 16-year-old Zhang Kaiyin won the fifth in the National City Sanda Competition, later he learned from the coach Liu Haike in Tagou Martial Arts School. Zhang Kaiyin was enrolled in Henan Sanda Group in 2004, and he was the $75 \mathrm{~kg}$ weightlifting champion of National Youth Sanda Competition in the same year. In 2007, he represented the Chinese team to participate in the "Iran President Cup" Sanda competition for the first time, and won the $80 \mathrm{~kg}$ weightlifting champion. In the same year Zhang Kaiyin won the $75 \mathrm{~kg}$ weightlifting champion in Sino Japanese Sanda Competition and Chongqing Worldwide Sanda Tournament. In 2010, Zhang Kaiyin triumphed over Thailand players and became the king of kings with absolute and undisputed advantage in China and Thailand Boxing Match. In 2011, he won the $75 \mathrm{~kg}$ weightlifting champion in National Men's Sanda Championship and WMA Star Game. In 2013, He won the $75 \mathrm{~kg}$ weightlifting gold medal for the Chinese team in the twelfth World Wushu Championships. Over ten years Zhang Sanda experience, which helps accumulates a stable and solid combat experience, and also pushes his sports career to the peak period.

\section{Zhang Kaiyin's winning characteristics of techniques and tactics in Wushu Sanda Competition}

Boxing features. In Wushu Sanda Competition, boxing is one of the most basic techniques, including straight punch, swings, hook and whip fist. The boxing of Sanda characterized by fast speed and flexible, it achieves the attack and counterattack through the combination of fists, fist and leg, or any mix other applicable tactics. The clever use of boxing directs a great threat to the opponent, whether the boxing is used properly in the whole competition, which directly determines the outcome of the athletes. We found that Zhang Kaiyin mainly uses the straight punch and swings, he rarely uses the hook and whip fist by observing Zhang Kaiyin's competition video of 2014 World Cup Wushu Sanda Competition. His fist is fast, accurate, his movement is flexible, and the 
overall success rate is high. From the competition video we can see that Kaiyin is good at seizing opportunities, he can skillfully uses Sanda skills and win the competition to show his personal style of boxing (as illustrated in Table 1).

Table 1.The personal characteristics of Zhang Kaiyin's boxing

\begin{tabular}{|c|l|}
\hline Classification of Boxing & Specific Characteristics \\
\hline Straight Punch & $\begin{array}{l}\text { The frequent use of straight punch, with fast speed, big } \\
\text { power, high flexibility, the success rate is as high as 70\% }\end{array}$ \\
\hline Swing & $\begin{array}{l}\text { The use of swing boxing accounted for 30\%, which mainly } \\
\text { are forehand swings,almost no back swing }\end{array}$ \\
\hline Hook and Whip Fist & $\begin{array}{l}\text { Rare use of hook and whip fist, once he used, it must with } \\
\text { has satisfactory effect }\end{array}$ \\
\hline
\end{tabular}

Leg features. Leg is the main attack skill in Wushu Sanda and it is also the most powerful method in combat and competition. In the Wushu Sanda Competition, whip leg, kick, side kick are the most common for Zhang Kaiyin. Zhang Kaiyin mostly uses the whip leg, and the low whip leg is his scoring weapon. Zhang Kaiyin takes the kicking as offensive start action which can block the opponent's attack, it is obvious that he has enough experience in the use of the leg, thus he can innovate on the basis of proficient use of legs (as illustrated in Table 2).

Table 2.The personal characteristics of Zhang Kaiyin’s kicking

\begin{tabular}{|c|l|}
\hline $\begin{array}{c}\text { Classification of } \\
\text { Kicking }\end{array}$ & Specific Characteristics \\
\hline Kick & $\begin{array}{l}\text { Zhang Kaiyin opens game with kicking, his legs are fast with high hit } \\
\text { rate, and the use frequency is close to 30\%. }\end{array}$ \\
\hline Lower Whip Leg & $\begin{array}{l}\text { In the game, the use of lower whip leg frequency is close to 50\%, and } \\
\text { the front whip leg has short attack route and fast speed, so he can use } \\
\text { it freely and retreat quickly. }\end{array}$ \\
\hline Higher Whip Leg & $\begin{array}{l}\text { The higher whip leg has a larger swing than the lower leg, the } \\
\text { moving line is longer, the requirement for the stability gravity is } \\
\text { higher, and the frequency of the use is low. }\end{array}$ \\
\hline
\end{tabular}

\section{The technical and tactical characteristics of Zhang Kaiyin's Wushu Sanda championship}

If you want to get the effective scores in Sanda competition, in addition to pay attention to kicking and boxing, the use of tactics is very necessary. In the martial arts competition, Zhang Kaiyin usually takes successive attack after hitting the opponent, and it is fast and accurate. He is proficient in boxing and kicking, sometimes he attacks and withdraws, sometimes he follows up to attack, and he has a keen judgement, which is because that Zhang Kaiyin is proficient in using the skills in peacetime training.

Analysis of psychological factors. Zhang Kaiyin's wining is not one-sided in Wushu Sanda Competition, it is closely related to daily training that he received, it also cannot separates from his good psychological quality that formed in a long-term battles. The good psychological qualities like enterprising spirit of martial arts, confident and calm challenge attitude, unyielding faith after twists and turns, and he is good at grasping the opportunity of in a changing environment, which are the fundamental reason for Zhang Kaiyin's wining.

Enterprising spirit of martial arts. Zhang Kaiyin's success in Wushu Sanda Competition is closely related with his efforts. Long term good training lays the foundation for Wushu Sanda, mature psychology is his magic weapon to win. Zhang Kaiyin loves martial arts since he was a child, he learned was martial arts in a formal school at the age of 13, he firstly practices the routine, then he began to learn Sanda. Zhang Kaiyin puts the traditional martial arts and Modern Sanda into a perfect combination, and he is flexible in competition and has a lower whip leg stunt. Zhang Kaiyin unceasingly enterprising spirit of martial arts makes he can use a beautiful set of Wushu to highlight his traditional Kung Fu, but it doesn’t affect his real fighting with the modern fighting 
philosophy. Zhang Kaiyin transferred from the traditional Sanda to the Modern boxing, which cannot do without his enterprising spirit of martial arts.

Confident and calm attitude. Different from some other Chinese Sanda athletes, who like negative fighting and defense, and rarely take the initiative to attack, Zhang Kaiyin has never been conservative, negative response to the game, which is his wisdom. Whether facing LamsongkramChuwatana or Wayne, Zhang Kaiyin is confident, calmly faced with the challenges, he won the opponent on the momentum, which is the basic characteristic held by all international superstar. Zhang Kaiyin has a characteristic of sworn, supremely confident in Wushu Sanda Competition, and a lot of domestic outstanding Sanda athletes are lack of this important characteristic.

Unyielding faith after twists and turns. Success is always ups and downs, it is the common characteristic of all the athletes, Zhang Kaiyin experienced the ups and downs before the winning in Sanda competition. Wayne defeated Thai boxing king Yodsanklai Fairtex and won the title of "King of the World Thai Boxing”. Wayne narrowly won Yodsanklai Fairtex and became the king of Thai boxing, thus Zhang Kaiyin and Yodsanklai Fairtex are more likely to win, which has become the most popular topic in that year, but this matchup ultimately failed to achieve. There are many reasons, and one of the most important reasons is that Zhang Kaiyin is a Sanda player within the system, so his leadership must give priority to the main task of the players, namely the planned game within the system. Zhang Kaiyin wants to play, but leadership didn't approve. Although Zhang Kaiyin participated in various businesses wrestling game over those years, there is no longer a classic battle, and his opponents no longer appear too like LamsongkramChuwatana and Wayne, and the personal value of Zhang Kaiyin also fails to get fully reflected. Since then, with the retirement from the professional team, his career in the system is ended, and the longer career out of the system is coming. Nowadays, he takes the advantage of his reputation in China, and he began to be incorporated into the system of $\mathrm{K}-1$. There is the world's most famous standing fighting game platform, where Zhang Kaiyin expresses his original suppressed value. Reviewing the current status of Zhang Kaiyin, his value is still not fully reflected. Because Sanda fails to be present in the Olympic Game, Zhang Kaiyin is walking alone in his own road, and the unyielding faith will enable him to go further in the future.

\section{Conclusion}

Chinese Wushu Sanda has developed into a new period, which shows the overall strong momentum of development. At present, the theoretical study of Zhang Kaiyin's fist, leg and technique in Wushu Sanda Competition, which is conducive to the inheritance of Wushu Sanda skills and tactics, it provides experiential learning materials for the other Wushu Sanda athletes. The degree of internationalization of Wushu Sanda is still inadequate. Nowadays, Sanda has not entered the Olympic Games, Zhang Kaiyin has been unable to represent the identity of Chinese Sanda athletes to participate in the Olympic Games, which is the current challenges facing China's Wushu Sanda. As the quintessence of Chinese sports, Wushu Sanda is a focus of intangible cultural heritage protection, which needs the further development of heritors, thus to ensure that the excellent Sanda tactics can be inherited from generation to generation. In the future development, Wushu Sanda has to maintain a strong momentum of development if it develops towards the Olympic Games. The current goal is to protect the sports level of active athletes, strengthen the reserve forces of Sanda, which is the focus of the development of Chinese Wushu Sanda.

\section{References}

[1] Yin Haili. Amendment rules of Sanda attack combination technology of using status [D]. Beijing University of physical education, 2014 (2009).

[2] Niu Tiezhu. Sanda athletes Zhang Kaiyin boxing techniques characteristic analysis [J]. of contemporary sports science and technology, 2014 (32). 
[3] Li Ping. Chinese martial arts [J] Chinese sports newspaper headquarters, December 1, 2011.

[4] Zhuang Dong, Wu Yuhua. and boxing boxing using Sanda technology comparison of [J]. sports space, 2014 (01).

[5] Zhao Lianbao. Research on the application of straight punch in Sanda [J]. Chinese Market, 2013 (45). 